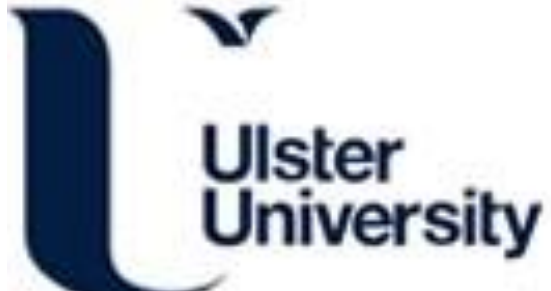

Review of Neuron Types in the Retina: Information Models for Neuroengineering

Valderrama, G., McGinnity, TM., Maguire, LP., \& Wu, Q. (2009). Review of Neuron Types in the Retina: Information Models for Neuroengineering. In Unknown Host Publication (Vol. 5768, pp. 335-344). Springer. https://doi.org/10.1007/978-3-642-04274-4_35

Link to publication record in Ulster University Research Portal

\section{Published in:}

Unknown Host Publication

Publication Status:

Published (in print/issue): 17/09/2009

DOI:

10.1007/978-3-642-04274-4_35

\section{Document Version}

Publisher's PDF, also known as Version of record

\section{General rights}

Copyright for the publications made accessible via Ulster University's Research Portal is retained by the author(s) and / or other copyright owners and it is a condition of accessing these publications that users recognise and abide by the legal requirements associated with these rights.

\section{Take down policy}

The Research Portal is Ulster University's institutional repository that provides access to Ulster's research outputs. Every effort has been made to ensure that content in the Research Portal does not infringe any person's rights, or applicable UK laws. If you discover content in the Research Portal that you believe breaches copyright or violates any law, please contact pure-support@ulster.ac.uk. 


\title{
Review of Neuron Types in the Retina: Information Models for Neuroengineering
}

\author{
German D. Valderrama-Gonzalez, T.M. McGinnity, \\ Liam Maguire, and QingXiang Wu \\ Intelligent Systems Research Centre, School of Computing and Intelligent Systems, \\ University of Ulster at Magee Campus, Derry, BT48 7JL, Northern Ireland, UK \\ http://isrc.ulster.ac.uk/
}

\begin{abstract}
Powerful information processing functions are performed in the mammalian retina in which basic units are different types of neurons. This paper presents the types of neurons and their roles in the visual processing system. The aim is to review the principles of how an artificial visual system could be constructed based on a comprehensive understanding of biological systems.
\end{abstract}

Keywords: Retina, cell types, neuroengineering, computational neuroscience, artificial visual systems.

\section{Introduction}

A challenging goal for engineers and computer scientists is to apply knowledge from biological research to create human-like intelligent artificial visual systems (AVS) [1, 2]. In order to achieve this goal, it is necessary to dissect the key principles that underpin biological vision $3,4,5,6$. The focus of this paper is to review the different types of neurons in the retina and their circuitry to process information. A summary of anatomy and physiology is presented along with some implications for modelling.

\section{A Review of the Biological Retina Cell Types}

Histologically, the retina is divided in 5 layers: (i) outer nuclear layer which contains the nucleus of the photoreceptors, (ii) the outer plexiform or interplexiform layer (IPL) where the synapses between photoreceptors, horizontals and bipolars take place, (iii) the inner nuclear IPL layer which contains the cells bodies of horizontal, bipolar and amacrine cells, collectively called interneurons, (iv) the inner IPL where the synapses between amacrine, bipolar and retinal ganglion cells (RGCs) take place and finally (v) the ganglion cell layer which contains the soma of the RGCs. The retina can be compared to a neural network (NN) of three layers (Fig. 17a) 7, 8 where the second and fourth histological layers are just connections. The outer nuclear layer where the photoreceptors are located corresponds to the NN input layer. The hidden layer of a NN is analogous to the

C. Alippi et al. (Eds.): ICANN 2009, Part I, LNCS 5768, pp. 335-344, 2009.

(C) Springer-Verlag Berlin Heidelberg 2009 
inner nuclear IPL that contains the nucleus of the interneurons. The ganglion cell layer is analogous to the NN output layer.

Photoreceptors are cells that respond to light by graded changes in the membrane potential. Photoreceptors have an hexagonal shape and an ON and OFF receptive field ( $\mathrm{RF}$ ) organisation [3]. There are two kinds of photoreceptors in the human retina: cones and rods. Rods are responsible for night vision, they detect dim light present at dusk or at night (moonlight). The rod system is highly sensitive to light, but is achromatic. Cones are responsible for day vision, they provide better spatial and temporal resolution, and mediate colour vision. Cones are densely packed in the centre of the retina, the fovea, thus providing the retina with a high resolution area in the centre; the surround of the retina has equilibrium but the periphery is vastly dominated by rods [1. On Figure 1. for visual simplification, cones are shown contacting a single bipolar cell; in reality they contact several bipolars of the same type and viceversa. The (L)ong or red cone and the (M)iddle wavelength-sensitive or green cone (Fig. 19) transmit "red-green colour opponency" signals to the midget ganglion cell (P-RGC) [9]. The short wavelength cone termed $\mathrm{S}$ or 'blue cone' has its own circuitry system (Fig. 11.). Chromatic information in a single channel tells the brain where along the spectrum from blue to red-green the stimulus lies (i.e. "blue-yellow colour opponency" signal).

Horizontal cell response is a function of the average intensity of stimulation within its RF. Horizontals enhance the concentric sensitivity of bipolar and RGCs, exciting or inhibiting surrounding photoreceptors. In mammals [9, 10, there are two types of horizontals: $\mathrm{H} 1$ and $\mathrm{H} 2$. L- and M-cones relay and receive feedback (inhibition) from H1 horizontals (Fig. 11d). H1 cells sum the L- and Mcone inputs to produce a "L-M-cone-contrast-gain" 9. H2-rods are far from the horizontal soma, making $\mathrm{H} 2$ cells electronically isolated from the cone's feedback system. Thus, rods have an independent feedback system driven by and fed by rods [10].

Bipolar cells participate in the straight forward flux of visual information or direct pathway as well as on the indirect or lateral pathway. If the direct pathway is excitatory, the indirect pathway is inhibitory and vice versa [4. Both pathways are integrated by the bipolar to generate a centre/surround or 'Mexican hat' RF. There are two size types of bipolars; the larger type innervates the rods ('rodbipolars') while the smaller type is connected to the cones ('cone-bipolars') [5]. The mammalian retina contains 9-11 types of cone-driven bipolars [5, 11] which subdivide into transient and sustained (Fig. 1b). Transient bipolar cells (b1) respond ON- and OFF- to high frequency light present anywhere in their RF. Sustained bipolars (b2/b3) which respond to low frequency light are also split on ON-sustained and OFF-sustained. This subdivision filters the signal into its temporal components, thus adding temporal filter behaviour to the retina [12]. 


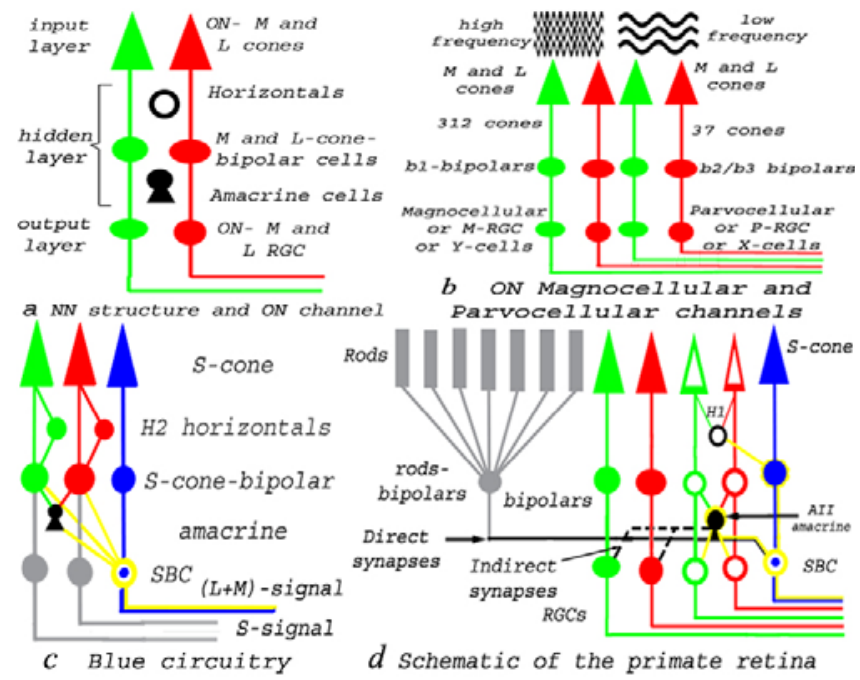

Fig. 1. This diagram shows the basic information processing channels in the primate retina and much detail is omitted. (a) The retina can be compared to a NN. Comparative chromatic information along the red to green spectrum is gathered in the (L-M)-channel. (b) ON version of the magnocellular and parvocellular channels. (c) Koniocellular pathway. (d) Rod circuitry. See text for details.

Amacrine cells: There are 20 morphologically different types of amacrines that use at least 8 different neurotransmitters. Some amacrines have similar functions to horizontals: they mediate antagonist inputs to RGCs from bipolars in the RGC's surround. Others have been implicated in shaping the complex RF properties of specific classes of RGCs. Amacrines also have two different types of response: transient and sustained. Rods and cones connect in parallel to separate bipolars, forming a separate cone and a rod circuitry. Rods' circuitry are simpler than cones (Fig. 11) [5]. At least two different pathways convey rod signals to RGCs in the moderately and extremely dark-adapted eye [4]. Hundreds of rods convey information to a single rod-driven bipolar synapsing on AII amacrine cell which give their output (a) directly to OFF-centre-RGCs and (b) indirectly to ON-centre-RGCs via cone-bipolars. Thus, the rod pathway attains access to the highly structured circuitry of the cone pathway, i.e. a directionally selective RGC maintains its function in dim light [4, 5].

Ganglion cells receive their input from bipolar and amacrine cells. They transform this information into APs that are the output of the retina. This is the first class of neurons in the visual pathways which actually uses APs to transmit information. Visual information includes light intensity (driven by rods) and colour information (driven by cones) [4. Their soma is located in the retina but 
their long axons form the optic nerve that ends at different nuclei in the thalamus, like the LGN. The neurons whose soma is at the LGN and axon travel to V1 in the visual cortex and are also called ganglion cells. A long-held principle is that each ganglion cell type projects information to the brain in distinct parallel pathways; however, not all ganglion types leads to a diferent pathway [5, 13. Three pathways are well known:

Magnocellular pathway: Some L-M-cones respond more vigorously to high frequency light. H1-horizontals biased those transient L-M cones. They both gather information to b1-bipolars and transient amacrines, which in turn pass it to M-RGC. M-RGCs are also called parasol cells, Y-ganglion cells in monkeys and $\alpha$-cells in cats $4,13,14$. M-RGCs are large neurons that have large concentric RFs which respond in a non-linear way with a transient response to temporal frequency modulation. M-RGCs mainly get input from the periphery of the retina and have a high degree of convergence. A single M-RGC receives input from approximately 100 rod-bipolars which in turn receive input from as many as 1500 rods and 8-10 cones-bipolars type b1 which in turn receive input from 312 cones [4,5]. This high convergence enhances sensitivity of the magnocellular channel. The high sensitivity (to light) of the rods, added to the high convergence provides a night vision channel highly responsive to movement. MRGCs project to the first and second layers of the LGN. There are two types of neurons on this channel: $\mathrm{ON}$ and $\mathrm{OFF}$.

Parvocellular pathway: Some other L-M-cones are more sensitive to low frequency light. They feed b2/b3-bipolars and sustained amacrines, which in turn biases P-RGC. P-RGCs are also called $\beta$-ganglion cells in cats, midget ganglion cells or X-ganglion cells in monkeys [4,13,14. P-RGCs are small, having a small linearly summing RF with a sustained response. P-RGCs are found nearer the fovea and are concerned with high resolution vision. In the fovea, P-RGCs constitute about $90 \%$ of all the RGCs whereas in the periphery they constitute only $40-45 \%$ [15. P-RGCs end at the Parvocelular $\left(3^{r d}-6^{\text {th }}\right)$ layers of the LGN. $\mathrm{P}-\mathrm{RGC}$ also comes in $\mathrm{ON}$ and $\mathrm{OFF}$ versions.

Koniocellular pathway: S-cones provide their ON-signal to their own specialised $([\mathrm{S}-\mathrm{ON} /(\mathrm{L}+\mathrm{M})-\mathrm{OFF}])$ bipolar cells. The $(\mathrm{L}+\mathrm{M})-\mathrm{OFF}$ signal is provided with a delay by surrounding cones that had been contacted by H2-horizontals [9, 16]. Specialised bipolars relay to a dedicated class of RGCs, the blue-ON/ yellow-OFF or $[\mathrm{S}-\mathrm{ON} /(\mathrm{L}+\mathrm{M})-\mathrm{OFF}]-\mathrm{RGC}$ or small bistratified cell $(\mathrm{SBC})[13$ 15, 17. Nonetheless, there is no agreement in the literature whether the OFF$(\mathrm{L}+\mathrm{M})$ signal circuitry is cones-H2-bipolars-SBC [9, 13, 17] or cones-bipolarsamacrine-SBC 18, or a mixture 19] (Fig. 15). Yet, another alternative is that H1-horizontals contact M-bipolars providing the (L+M)-OFF signal directly to SBC [9]. 


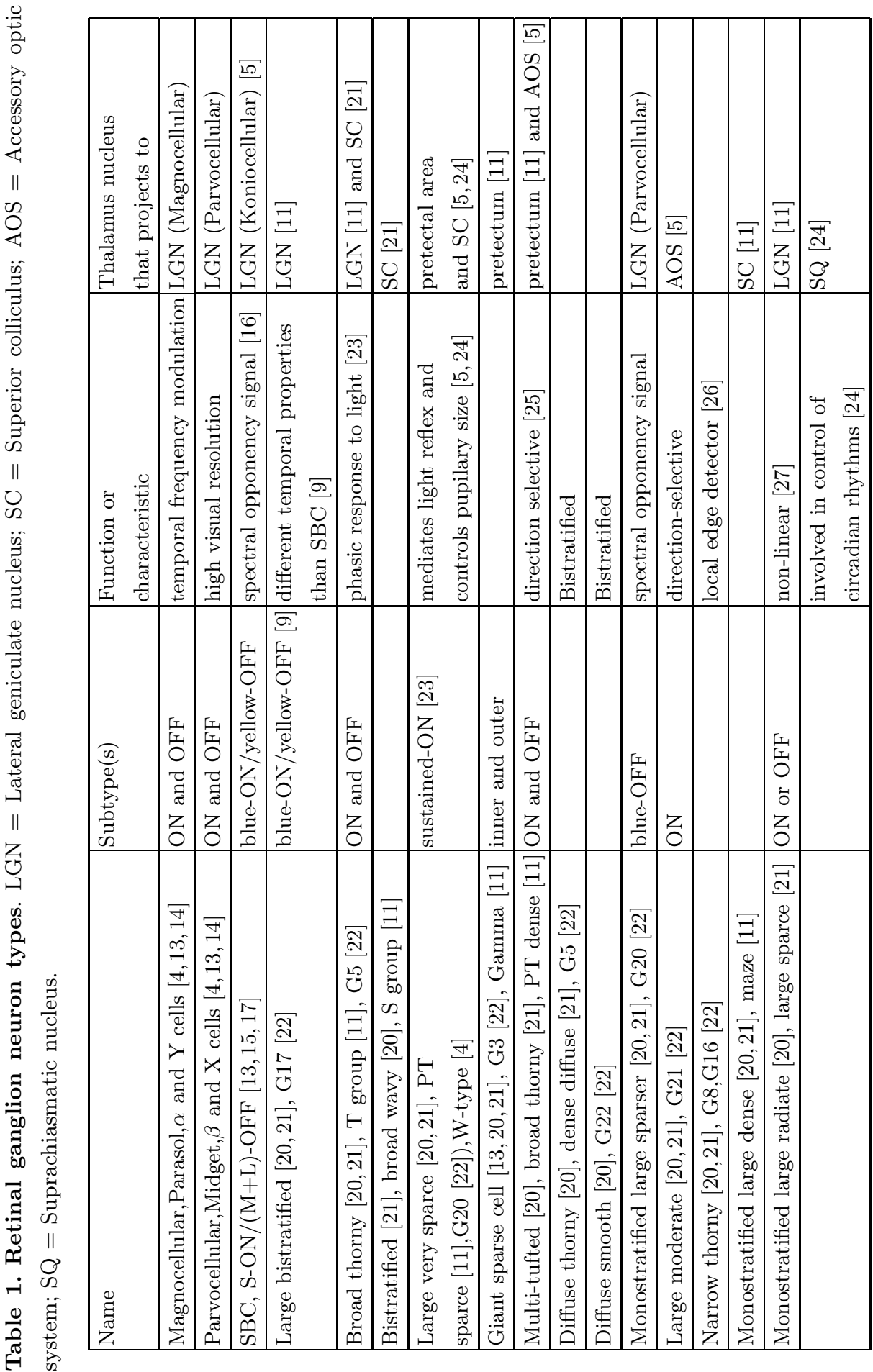


Extracellular recordings of APs from the optic nerve allow fairly easy visualisation of RGC's activity. Thanks to systematic anatomical studies (visually guided microinjection) and electrophysiology research in monkeys and humans 17-22 different types of RGC have been identified, as summarised in Table 1 .

\section{Implications for Modelling}

From the above review it is clear that visual information is processed in the retina by different types of neurons. Understanding the neurons circuitry and computations leads to understanding the information processing in the retina. The state-of the art of computational neuroscience of vision is that only a few types of neurons of the visual system are modelled. Therefore, we present a set of issues for simulation of an early stage of an AVS as follows: (1) What difficulties will we encounter when a visual computational model is used with multiple neuron types? Several issues can arise here, (a) Photoreceptors in biological systems are hexagonal, therefore a biologically inspired computational model should be arranged in a hexagonal grid [8]. However, there are no commercially available cameras that takes "himages" using hexagonal pixels or "hixels" rather than conventional square pixels. See 28] for a review on hexagonal image processing. (b) Also, the computational power required to do this processing in a biologically inspired way is huge and was not possible a decade ago. (c) In spite of great scientifical advances, our knowledge of the visual system neuron types, their connections and computations remain unclear. (2) Based on what is found on biology it is useful to assume that AVS would be more powerful if more types of neurons were considered in the models. For example, this review shows that the retina contains two forms of photosensitive neurons, i.e. rods and cones. Three types of cones have primary sensitivity of red, green, or blue light. By using three types of cones and rods for modelling of the visual system the colour processing mechanism of the retina in a wide variety of light conditions may be implemented in AVS. This mechanism is able to combine the gray-scale and RGB signals instead of only gray scale in current AVS models. (3) How can we use multiple neuron types in one visual system model? This can be achieved by oversampling the image and using a highly organised sampling architecture based on RFs and by implementing different channels each with particular neuron types that extract a specific feature from the image.

\section{Architectural Model}

Based on biological findings [6] it is possible to create early stage modelling of an AVS (Fig. 2). The initial stage of development of this model is the retina (encircled yellow) which is the focus of this paper. As shown in the diagram, the magnocellular pathway contributes to both the 'where' and the 'what' pathways where as the parvocellular mainly contributes to the 'what' pathway. On an early stage of modelling blue-cone signal can be transmited by the magnocellular and parvocellular pathways. Thus, leaving us with four channels: ON and OFF 


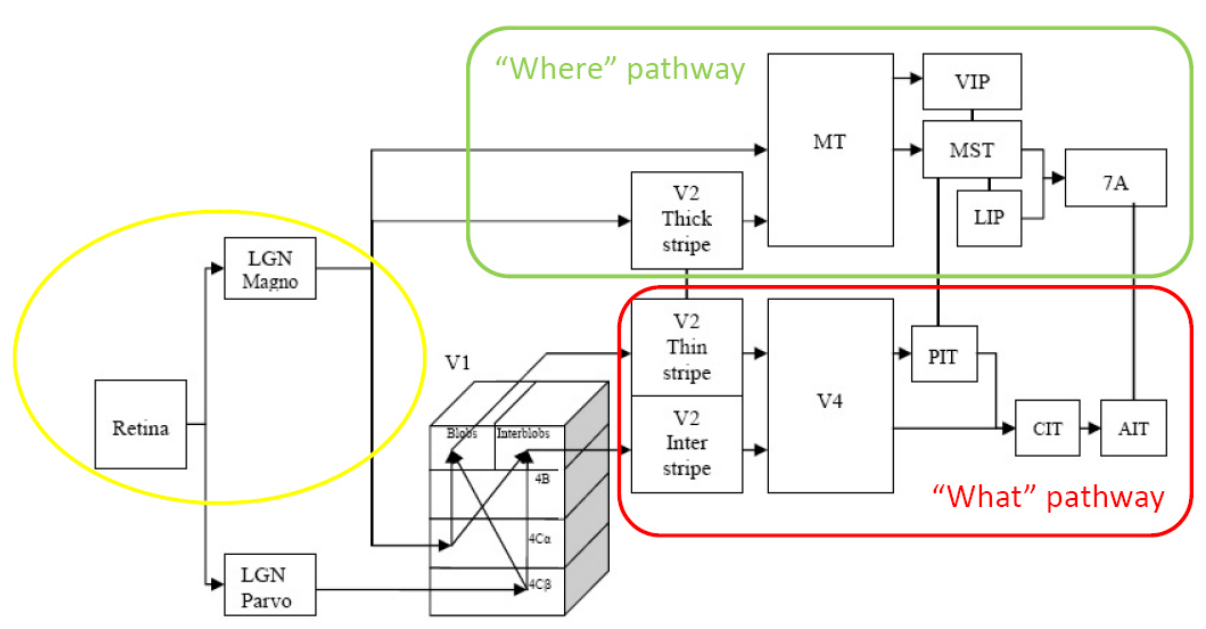

Fig. 2. Hierarchical organization of the overall proposed artificial visual system model. The Where pathway that performs motion detection and the What pathway that performs object recognition. Modified from [6].

magnocellular and $\mathrm{ON}$ and OFF parvocellular. Each of those channels relay red $(\mathrm{L})$, green $(\mathrm{M})$, blue $(\mathrm{S})$ and grey (rod) type of information. As an example the L-(ON and OFF)-magnocellular channels are described next. The L-ONMagnocellular-RGC processes an ON version of high frequency red light within its RF (Fig. 1b and Fig. 3a), and a L-OFF-Magnocellular-RGC processes the OFF version of the same signal (Fig. 1 d and Fig. 3r). Neighbouring RGC of the same type convey to a low hierarchy complex cell in the brain with a complex RF (Fig. 3b,d,e). Sequentially this cell conveys information to a higher level cell that compiles information regarding high frequency red light in the entire image (Fig. 3: ). Each RGC type processes specific information coming from a particular area (i.e. RF) in the visual field (i.e. image).

Now, how to combine those theoretical channels with a practical architectural model? An easy way to achieve this is to apply the following architectural model of the retina to each colour type of information. In this hypothetical architectural model the centre of each simple-RF is formed by 7 hexagonal 'hixels' and the surround is formed by two hexagonal rings of 'hixels' (Fig. [3a). ON-complex-RFs are composed of 7 hexagonal ON-simple-RFs (Fig. 3r). For the ON-Magnocellular channel when arranged to constitute the complex-RF, each simple-RF has six overlaping 'hixels'; thus, overlaping a single (strategically located) 'hixel' with each neighbour. Also note that there are 'hixels' that are not-included on the ON-Magnocellular-complex-RF arrangement (Fig. 3r). The non-including 'hixels' of the ON-Magnocellular complex-RFs are the centre of the OFF-Magnocellular complex-RFs (Fig. 3 d-f). As this 'hixel' was not included on the ON representation this means that it is not the same RF with inverted sign but rather another different channel. For the surrounding of the 


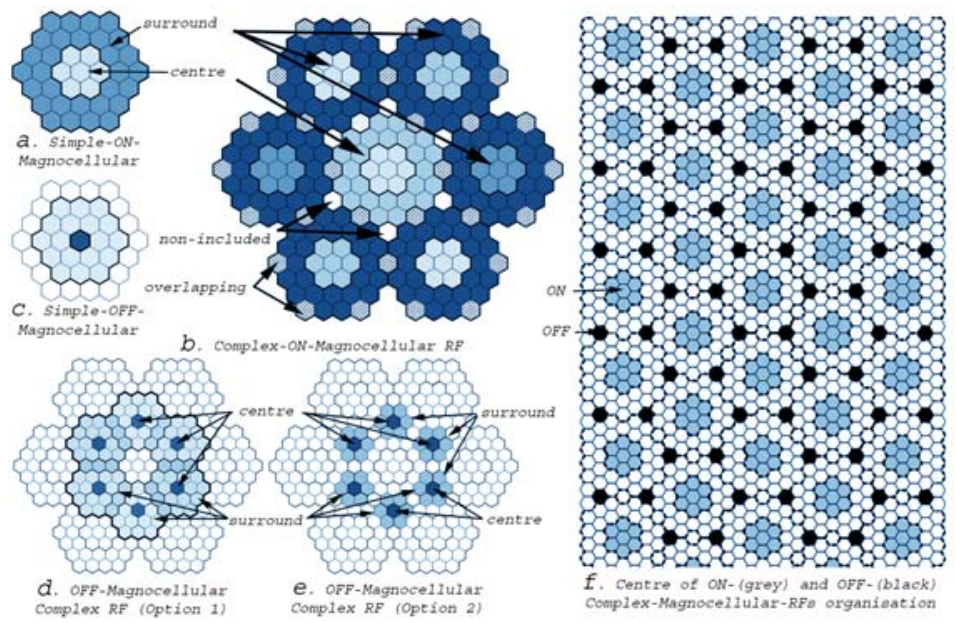

Fig. 3. Hypothetical model of Magnocellular simple- and complex-RFs. (a) ON-Simple and (b) ON-Complex Magnocellular-RF. The centre of this complex-RF is a simple-RF and the surround is drawn by six simple neighbouring RFs. (c) OFFSimple (for option 1) and (d-e) OFF-Complex Magnocellular-RF, see text. (f) Only the centres of ON-(blue) and OFF-(black) Complex-Magnocellular-RFs organisation are shown.

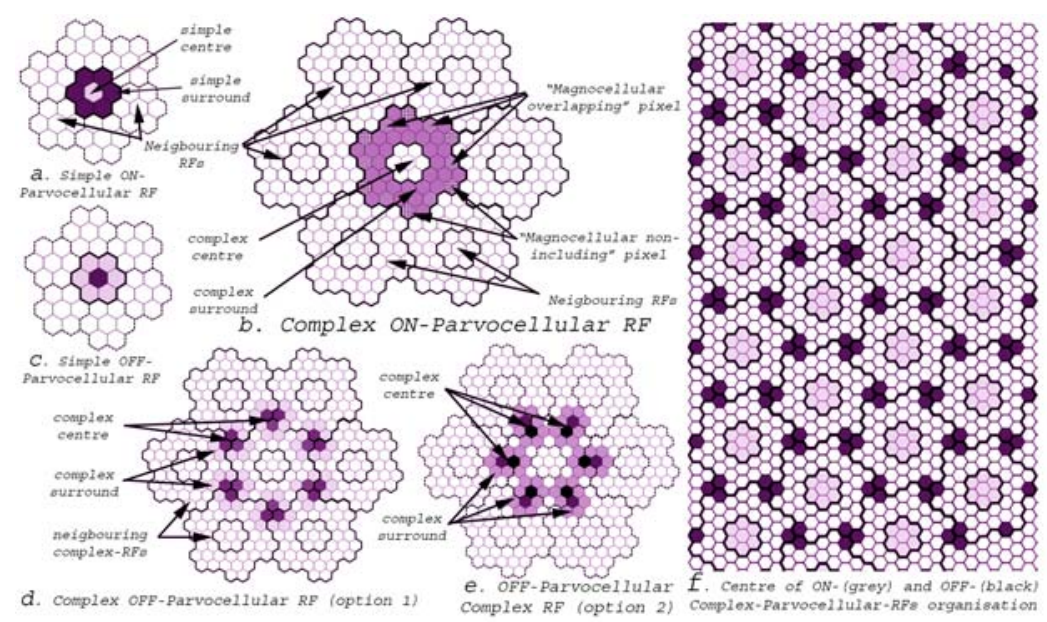

Fig. 4. Early stage model of simple and complex-Parvocellular-RFs. (a)ONsimple and (b) ON-Complex-Parvocellular-RF. (c) OFF- Simple-Parvocellular-RF. (d) The magnocellular's non-included-hixel is the centre of the OFF-ComplexParvocellular channel. (e) The magnocellular's overlapping-pixel is the centre of the OFF-Complex-Parvocellular channel. (f) Centre of ON-(purple) and OFF-(black) Complex-Parvocellular-RFs' organisation. 
OFF-Magnocellular complex-RF, there are two options whether an overlap is beneficial (option 1) or not (option 2). The first option is a big surrounding area formed by two rings of 'hixels' so that there is a continuity of the surrounding area (Fig. $3 \mathrm{~d}$ ). If this continuity that implies overlaping was undesirable an smaller surrounding area formed by just one 'hixel' ring would be more appropriated (Fig. 3e). In both cases, the centre of the ON-Magnocellular complex-RFs is respected (Fig. 3b). Thus, despite the overlapping in the surround on the selection of pixels (photoreceptors) there is no overlapping in the organisation of complex-RF. The centre of both ON- and OFF- Magnocellular channels are shown in Figure 3. Moreover, in this hypothetical model as in biology, the Parvocellular simple-RFs are smaller than their Magnocellular counterpart. For that reason the centre is just a 'hixel' and the surround is just a ring of six 'hixels' (Fig. 4a,c). The centre of the ON-Parvocellular complex RF, and the surround is shaped by six neighbouring simple-RFs (Fig. 4b). As for the OFF system, there are also two options. The first option is that the OFF-centre is outlined by the "Magnocellular's non-included" hixel (Fig. 4 $\mathrm{d}$ ). The second option is that the OFF-centre is delineated by the "Magnocellular's overlaping" hixel (Fig. 44). Both ON and OFF centres of the Parvocellular channel are displayed on Figure 4f. As presented here, the Parvocellular system has not overlapping at all.

\section{Conclusions}

The mammalian retina contains different neuron types and subtypes, each with a different function. Almost each RGC type has its own circuitry or pathway that processes a specific visual response property along the visual field. In each of those individual channels information gathered at photoreceptor level is relayed in purpose-specific cells that leads to specific feature extraction of the visual information. Consequencely, the retina output is composed of multiple parallel specific feature representations of the same image. Each of those individual specific pathways travels to a specific area in the visual cortex for higher processing. Thus, a promising way to model the retina is to model the individual channels in which each channel processes a specific feature. Therefore, a potential computational model of vision that solves those issues leads to future AVSs.

\section{References}

1. Bharath, A., Petrou, M.: Next Generation Artificial Vision Systems Reverse Engineering the Human Visual System. Artech House (2008)

2. Miikkulainen, R., Bednar, J.A., Choe, Y., Sirosh, J.: Computational Maps in the Visual Cortex. Springer, Heidelberg (2005)

3. Hubel, D.H.: Eye, Brain and Vision. Scientific American, New York (1988)

4. Rodieck, R.W.: The First Steps in Seeing. Sinauer associates, Sunderland (1998)

5. Masland, R.H.: The fundamental plan of the retina. Nat. Neurosci. 4, 877-886 (2001)

6. Merigan, W.H.: How parallel are the primate visual pathways? Annu. Rev. Neurosci. 16, 369-402 (1993) 
7. Lehky, S.R., Sejrtowski, T.J.: Network model of shape from shading: Neural function arises from both receptive and projective fields. Nature 333, 452-454 (1999)

8. Huang, C., Lin, C.: Bio-Inspired Computer Fovea Model Based on Hexagonal-Type Cellular Neural Network. IEEE Transactions on circuits and systems part 1 regular papers 54, 35-47 (2007)

9. Dacey, D.M.: Parallel Pathways for Spectral Coding in Primate Retina. Annual Reviews in Neuroscience 23, 743-775 (2000)

10. Wässle, H., Dacey, D.M., Haun, T., Haverkamp, S., Grünert, U., Boycott, B.B.: The mosaic of horizontal cells in the macaque monkey retina: With a comment on biplexiform ganglion cells. Vis. Neurosci. 17, 591-608 (2000)

11. Rodieck, R.W., Watanabe, M.: Survey of the morphology of the macaque retinal ganglion cells that project to the pretectum, superior colliculus and parvocellular laminae of the lateral geniculate nucleus. J. Comp. Neurol. 338, 289-303 (1993)

12. DeVries, S.H.: Bipolar Cells Use Kainate and AMPA Receptors to Filter Visual Information into Separate Channels. Neuron 28, 847-856 (2000)

13. Field, G.D., Chichilnisky, E.J.: Information processing in the primate retina: circuitry and coding. Annu. Rev. Neurosci. 30, 1-30 (2007)

14. Wässle, H., Boycott, B.B.: Functional architecture of the mammalian retina. Physiological Reviews 71, 447-470 (1991)

15. Dacey, D.M., Lee, B.B.: The'blue-on' opponent pathway in primate retina originates from a distinct bistratified ganglion cell type. Nature 367, 731-735 (1994)

16. Dacey, D., Peterson, B., Robinson, F.: Identification of an S-cone Opponent OFF Pathway in the Macaque Monkey Retina: Morphology, Physiology and Possible Circuitry. Invest. Ophthalmol. Vis. Sci. 43, 2983 (2002)

17. Kouyama, N., Marshak, D.: Bipolar cells specific for blue cones in the macaque retina. Journal of Neuroscience 12, 1233-1252 (1992)

18. Lukasiewicz, P.D.: Synaptic mechanisms that shape visual signaling at the inner retina. Prog. Brain Res. 147, 205-218 (2005)

19. Ichinose, T., Lukasiewicz, P.D.: Inner and outer retinal pathways both contribute to surround inhibition of salamander ganglion cells. J. Physiol. 565, 517-535 (2005)

20. Yamada, E.S., Bordt, A.S., Marshak, D.W.: Wide-field ganglion cells in macaque retinas. Vis. Neurosci. 22, 383-393 (2005)

21. Dacey, D.M., Peterson, B.B., Robinson, F.R., Gamlin, P.D.: Fireworks in the Primate Retina In Vitro Photodynamics Reveals Diverse LGN-Projecting Ganglion Cell Types. Neuron 37, 15-27 (2003)

22. Kolb, H., Linberg, K.A., Fisher, S.K.: Neurons of the human retina: A Golgi study. J. Comp. Neurol. 318, 147-187 (1992)

23. Isayama, T., Berson, D.M., Pu, M.: Theta ganglion cell type of the cat retina. J. Comp. Neurol. 417, 32-48 (2000)

24. Moore, R.Y., Speh, J.C., Card, J.P.: The retinohypothalamic tract originates from a distinct subset of retinal ganglion cells. J. Comp. Neurol. 352, 351-366 (1995)

25. $\mathrm{Pu}$, M., Berson, D.M., Pan, T.: Structure and function of retinal ganglion cells innervating the cat's geniculate wing: an in vitro study. Journal of Neuroscience 14, 4338-4358 (1994)

26. Amthor, F., Takahashi, E., Oyster, C.: Morphologies of rabbit retinal ganglion cells with complex receptive fields. Journal of Comparative Neurology 280, 97-121 (1989)

27. Kaplan, E., Shapley, R.M.: X and Y cells in the lateral geniculate nucleus of macaque monkeys. The Journal of Physiology 330, 125-143 (1982)

28. Middleton, L., Sivaswamy, J.: Hexagonal Image Processing: A Practical Approach. Springer, Heidelberg (2005) 